\title{
$\mathrm{T}_{\mathrm{THE}} \mathbf{A}_{\mathrm{SAN}} \boldsymbol{P}_{\mathrm{ACHIC}} \boldsymbol{S}_{\text {chour }}$ \\ MEDICAL AND HEALTH PROFESSIONS EDUCATION
}

\section{What drives attrition amongst obstetrics and gynaecology residents in Singapore?}

\author{
Jill Cheng Sim Lee ${ }^{1}$, Xiang Lee Jamie Kee ${ }^{2}$, Sharon Wiener-Ogilvie ${ }^{3}$, Bernard \\ Su Min Chern ${ }^{1,4}$ \& Chee Yang Chin ${ }^{5}$
}

${ }^{1}$ Division of Obstetrics and Gynaecology, KK Women's and Children's Hospital (KKH), Singapore; ${ }^{2}$ Yong Loo Lin School of Medicine, National University of Singapore (NUS), Singapore; ${ }^{3}$ NHS Education for Scotland, United Kingdom; ${ }^{4}$ SingHealth Duke-NUS Obstetrics and Gynaecology (OBGYN) Academic Clinical Programme, Singapore; ${ }^{5}$ Department of Cardiovascular Medicine, National Heart Centre Singapore (NHCS), Singapore

\begin{abstract}
Background: Resident attrition is costly but literature studying the motivations behind it in obstetrics and gynaecology (OBGYN) is lacking.

Objectives: We aimed to study the relationship between prior specialty-appropriate work experience and risk of attrition amongst OBGYN residents in Singapore, and identify factors placing residents at greater risk of attrition.

Methods: This nationwide mixed methods case control study studied all OBGYN residents in Singapore for the 2011 and 2012 intakes. A pre-piloted questionnaire was used to identify work experience and risk factors related to resident attrition. Structured interviews were conducted with a subgroup of participants chosen for diversity of educational background, work experience, and position towards residency.

Results: 28 of $33(84.8 \%)$ eligible residents participated in this study. Female (40.9\% vs. $0 \%)$, married $(50.0 \%$ vs. $22.2 \%)$ and Singaporean (38.1\% vs.14.3\%) residents more often considered attrition from training. Those accepted into residency after graduation were 33\% less likely to have considered leaving. No statistical difference was observed between residents with prior work experience and those without $(38.9 \%$ vs. $20.0 \%, \mathrm{p}=0.417)$. All interviewees believed that prior experience informs expectations and eases initial learning. Low job satisfaction related to mismatched expectations was the most quoted reason for considering attrition. $85.7 \%$ of interviewed residents were concerned about competing work and family demands.

Conclusions: Lack of specialty-appropriate work experience contributes to misinformed training expectations and increased attrition risk. Residents who have considered attrition may still desire career longevity in their specialty. Reduction in mismatched expectations promises to improve job satisfaction and translate to career longevity.
\end{abstract}

Keywords: $\quad$ Resident Attrition, Obstetrics and Gynaecology, Work Experience

\section{Practice Highlights}

- Resident training is expensive and attrition is stressful to the individual resident, training programme and health service.

- Recognition of risk factors for attrition from the training programme is important to improve identification of atrisk residents and take measures to reduce attrition.

- Mismatched expectations are often the reason for job attrition.

- Prior specialty-appropriate work experience may reduce misperceptions and better prepare residents for transition into the rigour of postgraduate specialty training.

\section{INTRODUCTION}

$20 \%$ of obstetrics and gynaecology (OBGYN) residents leave their training programme in the United States (US).(McAlister, Andriole, Brotherton, \& Jeffe, 2008) There are concerns that a similar pattern is followed in
Singapore where just two years into the adoption of a four-year American-styled residency training programme in 2011, a 5.9\% attrition rate (2 of 34) was observed amongst residents, with all departures voluntary. This figure is comparable to the $3.2 \%$ average annual attrition rate published by the largest residency 
sponsoring institution in Singapore across specialties. (Tamil Mannan et al., 2018).

Studies have postulated OBGYN attrition to be a result of workplace demands, such as the high litigation risk and long hours (S. E. Ogbonmwan \& D. E. Ogbonmwan, 2010). However, limited studies have formally investigated the motivations behind it. With the training cost for an OBGYN resident being estimated at US\$100,000 per year (American College of Obstetrics and Gynecologists, 2014), attrition is an exceedingly costly affair. Identifying factors behind resident attrition can potentially guide program decisions and efforts to retain trainees.

OBGYN, dermatology, paediatric medicine, ophthalmology, orthopaedic surgery, otorhinolaryngology and plastic surgery are seven specialties recognised by the Ministry of Health in Singapore to be highly subscribed specialties where additional training fees are paid by residents in these programmes. Between 2011 and 2018, the ratio of resident intake to applicants in OBGYN in Singapore ranged between 10 to $28.8 \%$ with a mean of $22.2 \%$. Recognising applicants at risk of attrition in these popular specialties may help improve resident selection criteria.

Furthermore, although previous work experience in OBGYN has been associated with more confidence in procedures (Ghaem-Maghami, Brockbank, \& Bridges, 2006) and is perceived to be useful in the eventual decision of specialisation (W. M. Rozen \& G. Rozen, 2006), literature behind the impact of residents' prior work experience on resident attrition is scarce. Given that in 2011 and 2012, approximately half of all applicants for OBGYN residency in Singapore were medical students with no prior work experience, the relationship between work experience and risk of attrition is potentially an important one.

Therefore, in this study, we aimed to identify factors within the individual resident which placed them at greater risk of attrition, as well as to elucidate the relationship between work experience and risk of attrition.

\section{METHODS}

A questionnaire was developed following discussion with institutional medical educators, faculty and resident peer leaders to identify relevant study variables with regards OBGYN resident attrition. The questionnaire was piloted twice on clinical and non-clinical healthcare personnel in order to ensure subject relevance.
Consideration of leaving the residency programme was used as a proxy for risk of attrition.

In Singapore, only two OBGYN residency programmes exist; SingHealth and National University Hospital Singapore. All OBGYN residents in both sponsoring institutions from the intake of 2011 and 2012 were invited to participate in this study $(\mathrm{N}=33)$. They were recruited over a 4-week period in 2014 during weekly national OBGYN teaching sessions and via email.

Questionnaire results were analysed using Fisher's exact test or independent sample t-test, where appropriate, and descriptive statistics. Gaps in explanations for observed trends were identified and questions addressing these gaps were subsequently developed for a structured faceto-face, individual interview conducted by a single investigator (JL) who is herself an OBGYN resident in Singapore and a resident representative elected by peers in this cohort. JL was excluded from this study cohort to reduce investigator bias.

A mixed pool of participants, comprising residents who had left residency, had considered leaving, or had never considered leaving, and of a variety of educational backgrounds and work experience were identified from the questionnaire and approached to take part in these structured interviews. 15 participants were approached for interview with $7(46.7 \%)$ agreeing to participate. Participants were also selected to have a mixture of gender, age, and marital status.

All interviews were taped and transcribed. Interview data was entered into QSR International NViVo 10 to allow line by line coding for emergence of themes based on the principles borrowed in part from grounded theory and triangulated with quantitative data obtained from the questionnaire.

In accordance with local practices, the study was exempted from SingHealth Centralised Institutional Review Board review. Participants were informed of the study requirements, timeline and aims. Verbal consent was then obtained and confidentiality was maintained throughout. Participation was strictly voluntary. Patients were not involved in this study and no funding was sourced or required for the conduct of this study.

\section{RESULTS}

\section{A. Questionnaires}

28 of $33(84.8 \%)$ residents agreed to participate in the study. Of these, 1 had already left OBGYN residency. The only other resident who had left withdrew her consent to participate due to reasons unrelated to this 
study. Tables 1 and 2 summarize the demographic data and characteristics of participants, respectively.

\begin{tabular}{|c|c|c|c|}
\hline Demographic data & $\begin{array}{c}\text { Total } \\
\mathrm{N}=28\end{array}$ & $\begin{array}{c}\text { Never Considered } \\
\text { Leaving } \\
\mathrm{N}=19\end{array}$ & $\begin{array}{c}\text { Considered } \\
\text { Leaving } \\
\mathrm{N}=9\end{array}$ \\
\hline \multirow[t]{2}{*}{ Mean Age (years) } & 29.0 & 29.3 & 28.6 \\
\hline & $\mathrm{N}$ & \multicolumn{2}{|c|}{$\mathrm{N}(\%)$} \\
\hline \multicolumn{4}{|l|}{ Gender } \\
\hline Male & 6 & $6(100)$ & $0(0)$ \\
\hline Female & 22 & $13(59.1)$ & $9(40.9)$ \\
\hline \multicolumn{4}{|l|}{ Marital status } \\
\hline Single & 18 & $14(77.8)$ & $4(22.2)$ \\
\hline Married & 10 & $5(50.0)$ & $5(50.0)$ \\
\hline \multicolumn{4}{|l|}{ Race } \\
\hline Chinese & 28 & $19(67.9)$ & $9(32.1)$ \\
\hline \multicolumn{4}{|l|}{ Nationality } \\
\hline Singaporean & 21 & $13(61.9)$ & $8(38.1)$ \\
\hline Non-Singaporean & 7 & $6(85.7)$ & $1(14.2)$ \\
\hline - Malaysian & 5 & $4(80.0)$ & $1(20.0)$ \\
\hline - $\quad$ Chinese & 2 & $2(100)$ & $0(0)$ \\
\hline \multicolumn{4}{|c|}{ Country of medical school } \\
\hline Singapore & 14 & $10(71.4)$ & $4(28.6)$ \\
\hline Outside of Singapore & 14 & $9(64.3)$ & $5(35.7)$ \\
\hline - United Kingdom & 9 & $5(55.6)$ & $4(44.4)$ \\
\hline - $\quad$ Malaysia & 2 & $1(50.0)$ & $1(50.0)$ \\
\hline - China & 2 & $2(100)$ & $0(0)$ \\
\hline - $\quad$ Australia & 1 & $1(100)$ & $0(0)$ \\
\hline
\end{tabular}

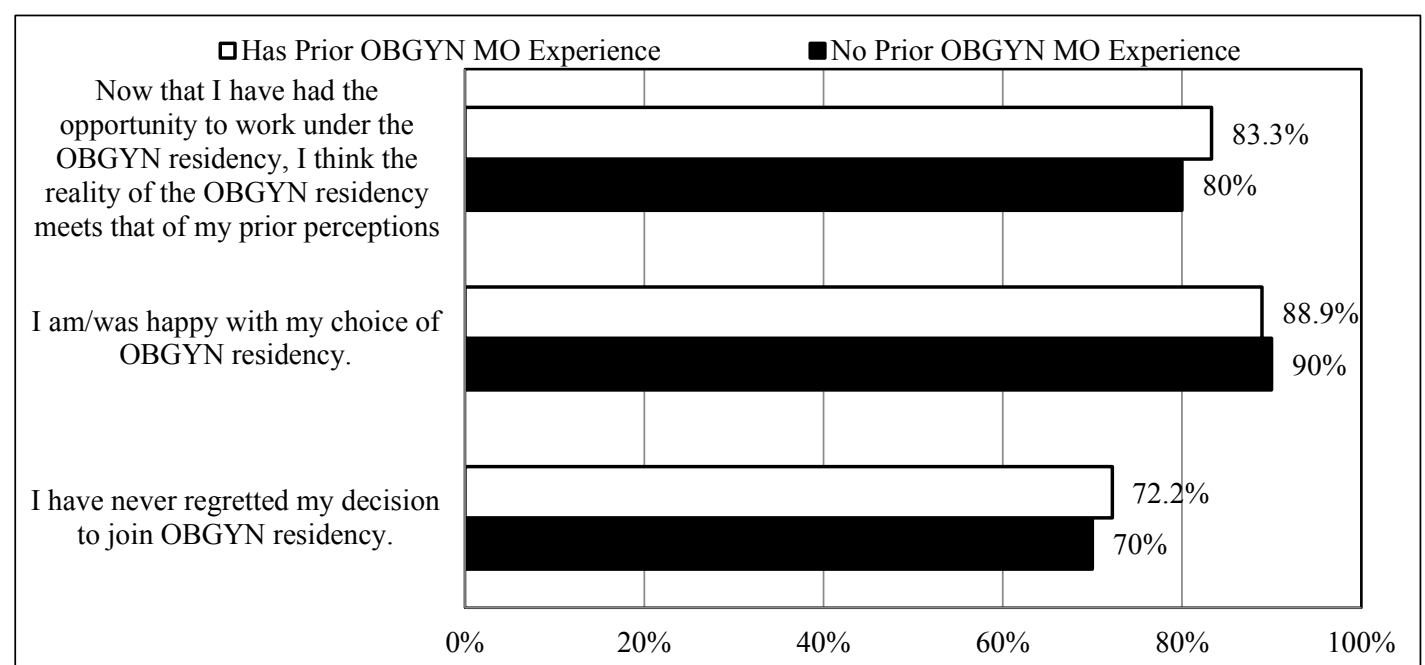

Figure 1. Influence of prior OBGYN MO experience on resident expectation, satisfaction and regret: Percentage positive responses (Agree or Strongly Agree)

$32.1 \%$ of residents surveyed had considered leaving the OBGYN residency programme. Female (40.9\% vs. $0 \%$ ), married $(50.0 \%$ vs. $22.2 \%)$ and Singaporean $(38.1 \%$ vs.14.3\%) residents appeared more likely to have considered leaving OBGYN training, although the differences did not amount to statistical significance. Country of medical school did not appear to make a difference to thoughts on attrition. 


\begin{tabular}{|c|c|c|c|}
\hline \multirow[t]{2}{*}{ Characteristics of Sample Data } & $\begin{array}{c}\text { Total } \\
\mathrm{N}=28\end{array}$ & $\begin{array}{c}\text { Never Considered } \\
\text { Leaving } \\
\mathrm{N}=19\end{array}$ & $\begin{array}{c}\text { Considered } \\
\text { Leaving } \\
\mathrm{N}=9\end{array}$ \\
\hline & $\mathrm{N}$ & $\mathrm{N}(\%)$ & \\
\hline \multicolumn{4}{|l|}{$\begin{array}{l}\text { Ranked Obstetrics \& Gynaecology } \\
\text { (OBGYN) as First Choice During } \\
\text { Residency Application }\end{array}$} \\
\hline Yes & 27 & $19(70.4)$ & $8(29.6)$ \\
\hline No & 1 & $0(0)$ & $1(100)$ \\
\hline \multicolumn{4}{|c|}{$\begin{array}{l}\text { Post-Graduate Year (PGY) at } \\
\text { Acceptance Into OBGYN Residency }\end{array}$} \\
\hline 0 & 7 & $4(57.1)$ & $3(42.9)$ \\
\hline 1 & 6 & $5(83.3)$ & $1(16.7)$ \\
\hline 2 & 9 & $6(66.7)$ & $3(33.3)$ \\
\hline 3 & 2 & $1(50.0)$ & $1(50.0)$ \\
\hline 4 & 3 & $2(66.7)$ & $1(33.3)$ \\
\hline \multicolumn{4}{|c|}{$\begin{array}{l}\text { OBGYN Medical Officer Experience } \\
\text { Prior to Entering OBGYN Residency }\end{array}$} \\
\hline Yes & 18 & $11(61.1)$ & $7(38.9)$ \\
\hline - $1-3$ months & 3 & $2(66.7)$ & $1(33.3)$ \\
\hline - $\quad 4-6$ months & 3 & $1(33.3)$ & $2(66.7)$ \\
\hline - $7-12$ months & 2 & $2(100)$ & $0(0)$ \\
\hline - $\quad$ 13-24 months & 6 & $5(83.3)$ & $1(16.7)$ \\
\hline - $\quad>24$ months & 1 & $0(0)$ & $1(100)$ \\
\hline - Unknown duration & 3 & $1(33.3)$ & $2(66.7)$ \\
\hline No & 10 & $8(80.0)$ & $2(20.0)$ \\
\hline
\end{tabular}

$42.9 \%$ of OBGYN residents who were accepted before graduation from medical school (postgraduate year [PGY] 0) had previously considered leaving OBGYN residency, while only $28.6 \%$ of OBGYN residents who were accepted post-graduation (PGY 1 and above) had previously considered leaving the programme. This suggested that those accepted after graduation were 33\% less likely to have considered leaving OBGYN residency in this cohort.

No statistical difference was observed between residents with prior work experience as a OBGYN Medical Officer (MO) compared with residents who had no such prior experience with regards to leaving residency ( $38.9 \%$ vs. $20.0 \%, \mathrm{p}=0.417)$. Notably, there were more participants in this study who had had prior work experience as an OBGYN MO than those who had no prior experience $(64.3 \%$ vs. $35.7 \%) .78 .6 \%$ of participants had prior OBGYN house officer (HO) experience. $30 \%$ of participants with no prior OBGYN MO experience also had no OBGYN HO experience prior to application to residency.

When assessed on a 5-point Likert scale about their prior expectations, current satisfaction levels, and feelings of regret towards the residency programme, there did not appear to be a discernible difference in responses between groups with prior work experience and those without (Figure 1).

\section{B. Structured interviews}

Table 3 describes the characteristics of interviewees. This study concentrated on themes that were common to all interviews.

\section{1) Prior work experience}

All interviewees felt prior OBGYN MO experience enables informed expectations and eases initial learning. OBGYN experiences as students or HOs were deemed not as insightful, providing only superficial specialtyspecific work.

Residents with prior experience as both an OBGYN HO and MO noted:

"The house officers' work scope is very different from that of a medical officer or resident."

"I think OBGYN is a very subspecialized specialty. It is not like internal medicine or general surgery where you probably have gained more experience as a medical student compared to OBGYN as a medical student. It is good to actually have a non-training medical officer posting, at least one or two (6-month) postings, before actually embarking on the residency programme to make sure that you are actually $O K$ to continue on with the 
residency programme. I know some of my classmates who had wanted to do OBGYN but after doing some postings here (in $O B G Y N$ ) they found that it was not their cup of tea. They are doing something else now and are happy with what they are doing right now."

Similarly, a resident with no prior OBGYN work experience noted:
"I think (prior medical officer experience) makes a difference because I think it makes a big difference for Year 1 and possibly a little bit in Year 2 because if you do not have any experience, then you are essentially catching up with everybody else because everybody else would have had experience. You spend a lot of time catching up and it may not be as easy to do well (in the programme) as compared to other people who have done OBGYN before."

\begin{tabular}{|c|c|c|c|c|c|c|c|c|c|}
\hline 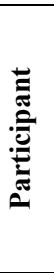 & 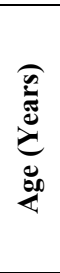 & 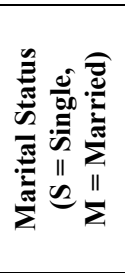 & 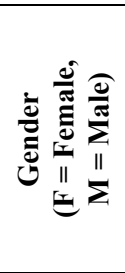 & 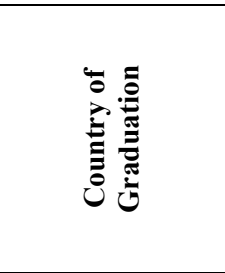 & 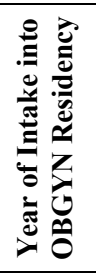 & 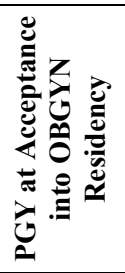 & 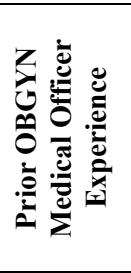 & 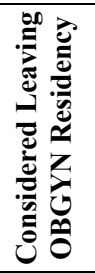 & 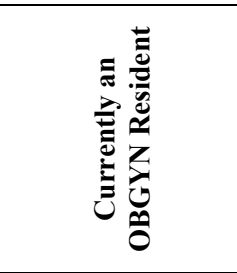 \\
\hline 1 & 28 & S & F & United Kingdom & 2012 & 2 & Yes & Yes & Yes \\
\hline 2 & 26 & M & $\mathrm{F}$ & Singapore & 2012 & 0 & No & Yes & Yes \\
\hline 3 & 28 & $\mathrm{~S}$ & $\mathrm{~F}$ & Singapore & 2011 & 2 & Yes & No & Yes \\
\hline 4 & 31 & M & M & United Kingdom & 2011 & 2 & No & No & Yes \\
\hline 5 & 31 & S & M & Singapore & 2011 & 3 & Yes & No & Yes \\
\hline 6 & 28 & M & $\mathrm{F}$ & United Kingdom & 2012 & 0 & No & Yes & Yes \\
\hline 7 & 29 & S & $\mathrm{F}$ & United Kingdom & 2011 & 1 & Yes & Yes & $\begin{array}{c}\text { No (Left in Year } 2 \\
\text { of Residency) }\end{array}$ \\
\hline
\end{tabular}

Table 3. Characteristics of interview participants

\section{2) Mismatched expectations}

Those considering attrition among the interview group most commonly attributed their reasons to low job satisfaction related to mismatched expectations of training.

This mismatch was often due to teething problems with a new residency programme. Reasons cited include "the [residency] system [not being] ready yet", as well as frustrations with "promised [...] opportunities" not perceived to be delivered, and thoughts of it "not yet [being] a well thought out programme".

Having undergone undergraduate medical training outside of Singapore also contributed to these unmet expectations, with one resident noting that "[...] training in $U K$ is different. The culture is all different. So, this isn't really what I expected."

Another echoed similar thoughts, saying:

"In the UK, when you become a consultant, your worklife balance actually improves. [...]So that's the perception (I had). (However,) the sad thing about $O B G Y N$ (in Singapore) is the work-life balance of a medical officer or resident is a lot better than (that of) a registrar or a consultant.'
These views were corroborated by locally trained residents. They perceived that the OBGYN residency was advantageous to graduates from Singaporean medical schools, most significantly in the earliest stages of residency training.

"People know you since probably medical school. It's easier to negotiate things and it is probably a better advantage if you are a local graduate to actually go on to do a subspecialty locally."

"The advantage that it confers is probably just initially during the whole settling in period with being a house officer and medical officer. I think the advantage levels off after a while and it's really just the initial familiarity that makes us a little bit more competent and efficient earlier on."

\section{3) Career aspirations}

Of note, most interviewees considering attrition still remained hopeful of long public service OBGYN careers, contingent on good leadership and collegiality.

One resident considering attrition stated:

"I think that having a positive work culture and happy work culture contributes a lot. I think I can settle for doing something...like for example I can work for less 
pay, and work longer hours and work harder for a good boss in a job that I truly feel satisfied in compared to having a cushier time but not being happy."

Another resident, also considering attrition, expressed:

"(In 10 years' time,) I don't really see myself going private. I think I'll probably just continue on, maybe in general OBGYN or doing admin or teaching."

\section{4) Balancing demands of work and family}

Almost all interview participants expressed concerns about balancing career and family in the future. Many, as illustrated earlier, recognised the increasing work demands expected of an individual as they progress up the OBGYN hierarchy which may threaten their ideals on work-life balance with many only "hoping to have a family with kid (and) some time for myself".

Others have observed and reported that:

"My friends are now struggling with family and life. Life means a balance; have time to go out for a meal, have time just to go out. At this moment, they are really struggling with work-life balance issues."

A male resident added that:

"In the next 10 years) I will probably want to get married and settle down with children and definitely want to pursue whatever I have learnt over the last 10 years. (I) will definitely continue my practice in $O B G Y N$. I have to support the family and be the breadwinner right?"

\section{DISCUSSION}

\section{A. Main Findings}

Similar to previous studies, a significant proportion of residents interviewed have considered leaving training. While no significant statistical differences emerged between the two groups ("never considered leaving" vs. "considered leaving"), certain patterns were observed.

This study recognised that residents who were female, married and Singaporean citizens trended towards considering attrition more often than their other counterparts. Despite no statistical significance identified between residents with prior specialty-specific work experience, qualitative data found that all interview participants agreed that such prior experience, especially at a level beyond that of a junior house officer was helpful to better manage their expectations of the training programme as well as eased their initial learning. With mismatched expectations leading to low job satisfaction, it is important to address training expectations prior to entry into OBGYN residency through opportunities such as prior OBGYN medical officer work experience.

The Asia Pacific Scholar, Vol. 4 No. 1 / January 2019

Copyright (C) 2019 TAPS. All rights reserved.

\section{B. Strengths and Limitations}

This study included residents from all training institutions in Singapore with a participation rate of $84.8 \%$. The high participation rate allowed for the majority of opinions to be studied in this case-control study. As the structured interviews were conducted by a single investigator who was known to all participants, the data may be scrutinised for bias. However, being part of the OBGYN residency programme, JL had a deeper understanding of the complexities of the medical training system beyond that of an independent researcher, allowing her to generate deeper meaning when analysing the qualitative data in this study. Furthermore, her position as a trusted peer-elected representative and guarantee of privacy and anonymity allowed participants to share views forthrightly during the individual structured interviews. To reduce bias, a standardised series of questions developed following analysis of the written questionnaire were asked of all participants during the structured interview and findings from the qualitative interviews were triangulated with the questionnaire data.

We recognise that as Singapore is a small country with small intakes of residents per annum and with only 2 residents having left OBGYN training in this studied cohort, this study was not adequately powered to measure statistical significance between those who considered attrition and those who did not. However, addition of the qualitative component of this study aimed to overcome the limitations of the small resident numbers. In addition, the withdrawal of participation from one of the two residents who had left training resulted in loss of a valuable voice in this study but as over a third of residents have considered the idea of leaving training, the discussion of driving factors for attrition could still be explored thoroughly in this study.

\section{Interpretation}

Female and married residents appeared more likely to consider leaving residency, possibly due to expectations in the still traditional setting of a nuclear family in Singapore. In Aryeel's 1993 study of dual-earner Singaporean couples, there was significantly more burnout in wives than in husbands, with stressors stemming from both career and non-career life domains. With the secondary status of women deeply rooted in Singaporean culture, working women are still expected to perform a disproportionate portion of the household chores and childcare, including playing a major role in helping manage their child's daily schoolwork in the highly competitive Singaporean education system. It is possible that non-career stressors impact one's performance and enjoyment of work, thereby leading to workplace dissatisfaction, as evidenced by how work- 
family conflicts have been reported to be a significant positive antecedent of burnout (Bacharach, Bamberger, \& Conley, 1991).

Non-Singaporeans appeared less likely to consider attrition than their Singaporean counterparts. $25 \%$ of participants were non-Singaporean, a testament to international migration of healthcare professionals. The global phenomenon of migration has been increasingly observed over the last decades, especially from less developed countries to more developed countries. Multiple "push factors" such as low wages, poor working conditions and promotion possibilities, inadequate management support, heavy workloads, limited access to good technology, and even to medicines, have all been recognized as "push factors" motivating health workers to leave their home countries (Connell, Zurn, Stilwell, Awases, \& Braichet, 2007). The World Health Organization ranked the Singapore health system sixth for overall performance amongst its member states in The World Health Report 2000 (World Health Organization, 2000). Malaysia ranked $49^{\text {th }}$ while China trailed in $144^{\text {th }}$ place. For these non-Singaporean doctors, when selected for residency in a perceivably more successful healthcare system, they have strong motivation to succeed in their new country. This is in contrast with their local counterparts who may perceive such postgraduate training opportunities as a norm and view it more as an expected outcome than a privileged opportunity.

With no statistical difference observed between those with and without prior OBGYN MO experience with regards to attrition in this small study, the influence of work experience is more clearly explored through the qualitative data component. All participants expressed unanimously that prior specialty-specific work experience would be beneficial, irrespective of whether they themselves had gained such experience or not. The two dominant reasons that residents felt that prior experience was helpful were: (1) they felt the learning curve in the first year of OBGYN residency was steep and the prior experience would better prepare them for this hurdle, and (2) they felt it would help them form more realistic expectations and manage their own expectations of residency training and a OBGYN career. This finding challenges the current drive to accelerate postgraduate training and selection of specialty training following graduation from medical school as seen in programmes such as Modernising Medical Careers.

Resident attrition data from SingHealth cites programme mismatch as the leading cause of resident separation from training (Tamil Mannan et al., 2018). Acknowledging that mismatched expectations are a key contributor to attrition risk, it appears that prior work experience, by means of providing exposure and thereby the formation of realistic expectations could help to reduce attrition from unmet expectations. Furthermore, Oraman, Unakitan and Selen (2011) found that job satisfaction is greater if there is congruency between the expectation and the experience while Shields and Ward (2001) report a $65 \%$ higher probability of nurses intending to quit if they had low job satisfaction.

Crucially, considering attrition was not associated with diminished career aspirations. These residents should be identified early in training and provided with necessary support to continue to nurture dedicated clinicians with longevity in the healthcare service. A rigorous and supportive mentoring system utilizing the recognized strength of camaraderie and teamwork within the OBGYN residency could potentially sufficiently address this issue.

\section{CONCLUSION}

Lack of prior specialty-appropriate work experience is a major contributor to misinformed training expectations and increased inclination towards attrition. Prior specialty-appropriate medical officer experience appears to influence resident attrition as it reduces misperceptions and better prepare residents for the transition into the rigorous reality that is postgraduate specialty training. Reduction in mismatched expectations promises to improve job satisfaction and translate to career longevity. This should be aimed not just at the level of residents but also for retention of trained specialists within the healthcare service. Residents who have considered attrition may still desire career longevity in their specialty. Valuable early work experiences that prepare junior doctors for the demands of training are limited within contemporary training models that emphasize time-efficient specialty training. It is important that non-training medical officer posts continue to be made available to junior doctors to help bridge the expectation gap and transition of preresidency doctors into specialty training. Follow up research of this cohort upon completion of training will be useful to explore if factors that drive attrition change as the resident progresses through their training years and if prior pre-residency work experience indeed affects career longevity beyond specialty training on long-term follow up.

\section{Note on Contributors}

Dr. Jill Cheng Sim Lee is a senior resident in OBGYN at SingHealth and former Chief Resident for Education within her department. She has a Master of Science in Clinical Education and is involved in undergraduate 
medical education at Lee Kong Chian School of Medicine, Nanyang Technological University.

Miss Xiang Lee Jamie Kee is a final year medical student at Yong Loo Lin School of Medicine, NUS. She has a keen interest in medical education and is actively involved in running near-peer teaching initiatives at her university.

Dr. Sharon Wiener-Ogilvie is an associate adviser for quality and research in General Practice at NES with prior experience in public health and clinical governance. She holds an $\mathrm{MPH}$ and $\mathrm{a} \mathrm{PhD}$ and has published extensively in medical education, particularly around career choice, and evaluation of educational programmes.

A/Prof. Bernard Su Min Chern is Chairman of Division of OBGYN and Head and Senior Consultant of the Department of Minimally Invasive Surgery at KKH. He is also Chairman of the SingHealth-Duke-NUS OBGYN $\mathrm{ACP}$ and was formerly Programme Director of the SingHealth OBGYN Residency Programme.

Dr. Chee Yang Chin is a Consultant Cardiologist at NHCS with a sub-specialty interest in interventional cardiology. He has a Master of Science in Clinical Education and is Core Faculty to both the SingHealth Internal Medicine Residency Programme and Cardiology Senior Residency Programme.

\section{Ethical Approval}

In accordance with local practices, the study was exempted from SingHealth Centralized Institutional Review Board review.

\section{Acknowledgements}

The authors would like to acknowledge the support and cooperation provided by the residents, faculty and staff at the SingHealth OBGYN Residency Programme during the period of this study. We would also like to thank Ms Gillian Aitken and Dr Michael Ross from the University of Edinburgh for their advice during design and development phases of this study.

\section{Funding}

This is an unfunded study.

\section{Declaration of Interest}

All authors have no potential conflicts of interest.

\section{References}

American College of Obstetricians and Gynecologists (ACOG) (2014). Support the Training of Tomorrow's Doctors Today Act of 2013. Retrieved from

http://www.acog.org/ /media/Departments/Government\%20Relat ions\%20and\%20Outreach/2014CLCGMEAsk.pdf?dmc $=1 \&$ ts $=20$ $140327 \mathrm{~T} 1032213142$.

Aryeel, S. (1993). Dual-earner couples in Singapore: An examination of work and nonwork sources of their experienced burnout. Human Relations, 46(12), 1441-1468. http://doi.org/10.1177/001872679304601205.

Bacharach, S. B., Bamberger, P., \& Conley, S. (1991). Work-home conflict among nurses and engineers: Mediating the impact of role stress on burnout and satisfaction at work. Journal of Organizational Behaviour, 12(1), 39-53. http://doi.org/10.1002/job.4030120104.

Connell, J., Zurn, P., Stilwell, B., Awases, M., \& Braichet, J. M. (2007). Sub-Saharan Africa: beyond the health worker migration crisis? Social Science \& Medicine, 64(9), 1876-1891. http://doi.org/10.1016/j.socscimed.2006.12.013.

Ghaem-Maghami, S., Brockbank, E., \& Bridges, J. (2006). Survey of surgical experience during training in obstetrics and gynaecology in the UK. Journal of Obstetrics and Gynaecology, 26(4), 297-301. http://doi.org/10.1080/01443610600594740.

McAlister, R. P., Andriole, D. A., Brotherton, S. E., \& Jeffe, D. B. (2008). Attrition in residents entering US obstetrics and gynecology residencies: analysis of National GME Census data. American Journal of Obstetrics and Gynecology, 199(5), 574 e571-576. http://doi.org/10.1016/j.ajog.2008.06.081.

Ogbonmwan, S. E., \& Ogbonmwan, D. E. (2010). Recruitment and retention in obstetrics and gynaecology in the UK. British Journal of Hospital Medicine (Lond), 71(2), 103-105. http://doi.org/10.12968/hmed.2010.71.2.46490.

Oraman, Y., Unakitan, G., \& Selen, U. (2011). Measuring employee expectations in a strategic human resource management research: Job satisfaction. Procedia - Social and Behavioral Sciences, 24, 413-420.

http://dx.doi.org/10.1016/j.sbspro.2011.09.022.

Rozen, W. M., \& Rozen, G. (2006). Postgraduate obstetrics and gynaecology experience: The role for early exposure. Australian and New Zealand Journal of Obstetrics and Gynaecology, 46(6), 538-540. http://doi.org/10.1111/j.1479-828X.2006.00656.x.

Shields, M. A., \& Ward, M. (2001). Improving nurse retention in the National Health Service in England: The impact of job satisfaction on intentions to quit. Journal of Health Economics, 20(5), 677-701.

Tamil Mannan, S. D., Heng, J. M. J., Andrada, J. A., Choon, P. H., Yeo, H. S. H. \& Lim, B. L. (2018, March). Improving resident selection through analysis of attrition rate in SingHealth Residency. Poster presented at the Accreditation Council for Graduate Medical Education Annual Education Conference, Orlando, FL.

World Health Organization. (2000). The World Health Report 2000: Health Systems: Improving Performance. Retrieved from www.who.int/whr/2000/en/whr00_en.pdf.

*Dr Jill C. S. Lee

Email: jill.lee.c.s.@singhealth.com.sg

Division of Obstetrics and Gynaecology

KK Women's and Children's Hospital

100 Bukit Timah Road, Singapore 229899

Tel: +6562255554 